EESTI NSV TEADUSTE AKADEEMIA TOIMETISED, 25. KOIDE FUUSIKA * MATEMAATIKA. 1976, NR. 2

ИЗВЕСТИЯ АКАДЕМИИ НАУК ЭСТОНСКОИ ССР. ТОМ 25 ФИЗИКА * МАТЕМАТИКА, 1976, № 2

удК $518: .517 .392$

Ю. ГИРШОВИЧ

\title{
ОДИН МЕТОД ПОСТРОЕНИЯ НАИЛУЧШИХ КВАДРАТУРНЫХ ФОРМУЛ ТИПА МАРКОВА
}

I. GIRSOVITS. PARIMATE MARKOVI TOOPI KVADRATUURVALEMITE TULETAMISE MEETOD Y. GIRSHOVICH. METHOD OF CONSTRUCTION OF MARKOV'S TYPE OPTIMAL QUADRATURE FORMULAS

Пусть $W^{(r)} L_{q}$ обозначает множество всех функций $f(x)$, которые на отрезке $[0,1]$ имеют абсолютно непрерывную производную порядка $r-1$ и удовлетворяют условию $\left\|f^{(r)}\right\|_{L q(0,1)} \leqslant M(M>0$ и $q(1 \leqslant q \leqslant \infty)$ - заданные числа).

Функционалы

$$
U_{i}(f)=\sum_{j=0}^{r-1}\left[\alpha_{i j} f^{(j)}(0)+\beta_{i j} f^{(j)}(1)\right] \quad(i=1, \ldots, 2 r),
$$

где $\alpha_{i j}, \beta_{i j}-$ заданные числа, считаем линейно независимыми.

Через $\bar{W}_{U}^{(r)} L_{q}$ обозначим множество всех функций $f(x)$, которые принадлежат множеству $W^{(r)} L_{q}$ и удовлетворяют условиям

$$
U_{i}(f)=0 \quad(i=1, \ldots, m),
$$

где $m(0 \leqslant m \leqslant 2 r)$ - заданное число. При $m=0$ это множество совпадает с $W^{(r)} L_{q}$.

Рассмотрим на множестве $\bar{W}_{U}^{(r)} L_{q}$ квадратурные формулы вида

$$
\int_{0}^{1} f(x) d x=\sum_{k=1}^{n} \sum_{j=0}^{\rho_{k}} B_{k j} f^{(j)}\left(u_{k}\right)+\sum_{i=m+1}^{s} b_{i} U_{i}(f)+\bar{R}_{n}(f),
$$

где $0<u_{1}<\ldots<u_{n}<1$, а числа $n, s(m \leqslant s \leqslant 2 r), \quad \varrho_{k}\left(0 \leqslant \mathrm{Q}_{k} \leqslant\right.$ $\leqslant r-1)$ заданы. Формулы (2) назовем формулами типа Маркова.

Нас будет интересовать построение наилучшей $\left[{ }^{1}\right]$ на множестве $\bar{W}_{U}^{(r)} L_{q}$ формулы (2), т. е. той формулы (2), для которой величинӑ

$$
\bar{R}_{n}=\sup _{f \in \bar{W}_{U}^{(r)} L_{q}}\left|\bar{R}_{n}(f)\right|
$$

принимает наименьшее значение. 
Через $W_{U}^{(r)} L_{q}$ обозначим множество всех функций $f(x)$, которые принадлежат множеству $\bar{W}_{U}^{(r)} L_{q}$ и удовлетворяют условиям

$$
U_{i}(f)=0 \quad(i=m+1, \ldots, s) .
$$

Через $V_{1}, \ldots, V_{2 r}$ обозначим функционалы краевых условий, сопряженных к условиям (1) относительно дифференциального выражения $y^{(r)}\left[{ }^{2}\right]$, а через $K_{r, \rho}(V)$ - множество всех моносплайнов $K(x)$ вида

$$
K(x)=\frac{x^{r}}{r !}+\sum_{j=0}^{r-1} c_{0, j} x^{r-j-1}+\sum_{k=1}^{n} \sum_{j=0}^{\rho_{k}} c_{k j}\left(x-x_{k}\right)_{+}^{r-j-1}
$$

(где $u_{+}^{j}=u^{j}$ при $u \geqslant 0, u_{+}^{j}=0$ при $u<0$ ), удовлетворяющих условиям

$$
V_{i}(K)=0 \quad(i=1, \ldots, 2 r-s) .
$$

В [ $\left.{ }^{3}\right]$ доказано, что наилучшая на множестве $W_{U}^{(r)} L_{q}$ квадратурная формула вида

$$
\int_{0}^{1} f(x) d x=\sum_{k=1}^{n} \sum_{j=0}^{\rho_{k}} A_{k j} f^{(j)}\left(x_{k}\right)+R_{n}(f)
$$

однозначно определяется через моносплайн $K^{*}(x)$ наименьшего уклонения от нуля на отрезке $[0,1]$ в метрике $L_{p}\left(p^{-1}+q^{-1}=1\right)$ среди всех моносплайнов из множества $K_{r, \rho}(V)$, при этом

$$
\begin{gathered}
A_{k j}=(-1)^{j}\left[K^{*(r-j-1)}\left(x_{k}-0\right)-K^{*(r-j-1)}\left(x_{k}+0\right)\right] \\
\left(j=0, \ldots, Q_{k} ; k=1, \ldots, n\right), \\
R_{n}=\sup _{\operatorname{seW}_{U}^{(r)} L_{q}}\left|R_{n}(f)\right|=M\left\|K^{*}(x)\right\|_{L_{p}(0,1)},
\end{gathered}
$$

где $x_{k}$ - узлы, $A_{k j}$ - веса, $R_{n}$ - точная оценка ошибки наилучшей на множестве $W_{U}^{(r)} L_{q}$ формулы (4).

Т е о р е а 1. Для того чтобы формула (2) с узлами $u_{k}(k=1, \ldots, n)$, весами $B_{k j}\left(j=0, \ldots, \varrho_{k} ; k=1, \ldots, n\right), b_{i}(i=m+1, \ldots, s)$, точной оценкой ошибки $\bar{R}_{n}$ была наилучшей на множестве $\bar{W}_{U}^{(r)} L_{q}$, необходимо и достаточно, чтобы формула (4) с узлами $x_{k}=u_{k}$, весами $A_{k j}=B_{k j}$, точной оценкой ошибки $R_{n}$ была наилучшей на множестве $W_{U}^{(r)} L_{q}, \quad$ а веса $b_{i}$ вычислялись по'формуле

$$
b_{i}=V_{2 r+1-i}\left(K^{*}\right) \quad(i=m+1, \ldots, s),
$$

где $K^{*}(x)$ - соответствующий этой формуле моносплайн из $K_{r, \rho}(V)$. При этом $R_{n}=\bar{R}_{n}$.

Доказ а тельство. 1. Пусть формула (4) с узлами $x_{k}(k=$ $=1, \ldots, n)$ и весами $A_{k j}\left(j=0, \ldots, \mathrm{Q}_{k} ; k=1, \ldots, n\right)$ - наилучшая на множестве $W_{U}^{(r)} L_{q}, \quad R_{n}$ - точная оценка ее ошибки, $K^{*}(x)-$ соответствующий моносплайн из $K_{r, \rho}(V)$. В силу включения $W_{U}^{(r)} L_{q} \subset \bar{W}_{U}^{(r)} L_{q}$, а также того, что сужение на множество $W_{U}^{(r)} L_{q}$ любой формулы (2) совпадает с некоторой формулой (4), для любой формулы (2), в том числе и для наилучшей, справедливо неравенство

$$
R_{n} \leqslant \bar{R}_{n} .
$$


Интегрируя по частям, для любой функции $f(x) \in \bar{W}_{U}^{(r)} L_{q}$ (полагая $\left.x_{0}=1-x_{n+1}=0\right)$, имеем $\left[{ }^{2}\right]$

$$
\begin{aligned}
\int_{U}^{1} f(x) d x & =\sum_{k=1}^{n} \int_{x_{k}}^{x_{k+1}} f(x) K^{*}(x) d x= \\
& =\sum_{k=1}^{n} \sum_{j=0}^{r-1}(-1)^{j(j)}\left(x_{k}\right)\left[K^{*(r-j-1)}\left(x_{k}-0\right)-K^{*(r-j-1)}\left(x_{k}+0\right)\right]+ \\
& +\sum_{i=1}^{2 r} U_{i}(f) V_{2 r+1-i}\left(K^{*}\right)+(-1)^{r} \int_{0}^{1} f^{(r)}(x) K^{*}(x) d x .
\end{aligned}
$$

Если учесть, что

$K^{*(r-j-1)}\left(x_{k}-0\right)=K^{*(r-j-1)}\left(x_{k}+0\right) \quad\left(j=\varrho_{k}+1, \ldots, r-1 ; k=1, \ldots, n\right)$,

$U_{i}(f)=0 \quad(i=1, \ldots, m)$,

$V_{i}^{\prime}\left(K^{*}\right)=0 \quad(i=1, \ldots, s)$,

и положить в (7)

$$
\begin{aligned}
& u_{k}=x_{k} \quad(k=1, \ldots, n), \\
& B_{k j}=(-1)^{j}\left[K^{*(r-j-1)}\left(x_{k}-0\right)-K^{*(r-j-1)}\left(x_{k}+0\right)\right] \\
& b_{i}=V_{2 r+1-i}\left(K^{*}\right) \quad\left(i=m+\ldots, \mathrm{Q}_{k} ; k=1, \ldots, n\right),
\end{aligned}
$$

$\bar{R}_{n}(f)=(-1)^{r} \int_{0}^{1} f^{(r)}(x) K^{*}(x) d x$,

то получим формулу (2), для которой, в силу неравенства Гёльдера,

$$
\bar{R}_{n} \leqslant M\left\|K^{*}(x)\right\|_{L_{p}(0,1)}=R_{n} .
$$

Из (6) и (9) следует, что построенная формула (2) является наилучшей на множестве $\bar{W}_{U}^{(r)} L_{q}$, причем ((5) и (8))

$$
\begin{array}{ll}
u_{k}=x_{k} & (k=1, \ldots, n), \\
B_{k j}=A_{k j} & \left(j=0, \ldots, \varrho_{k} ; k=1, \ldots, n\right) .
\end{array}
$$

2. Пусть задана наилучшая на множестве $\bar{W}_{U}^{(r)} L_{q}$ формула (2) с точной оценкой ошибки $\bar{R}_{n}$. Если оценка ошибки наилучшей на множестве $W_{U}^{(r)} L_{q}$ формулы (4) $R_{n}$ удовлетворяет неравенству $R_{n}<\bar{R}_{n}$, то, согласно уже доказанной первой части теоремы, можно построить формулу (2), точная оценка ошибки которой на множестве $\bar{W}_{U}^{(r)} L_{q}$ есть $R_{n}$, что противоречит предположению. В то же время, положив в (4)

$$
\begin{array}{ll}
x_{k}=u_{k} & (k=1, \ldots, n), \\
A_{k j}=B_{k j} & \left(j=0, \ldots, \varrho_{k} ; k=1, \ldots, n\right),
\end{array}
$$

где $u_{k}-$ узлы, $B_{k j}$ - веса заданной наилучшей на множестве $\bar{W}_{u}^{(r)} L_{q}$ формулы (2), получим формулу (4), точная оценка ошибки которой есть $\bar{R}_{n}$. Эта формула (4) и является наилучшей на множестве $W_{U}^{(\tau)} L_{q}$, что доказывает теорему.

С лед с т в и е. Для построения наилучшей на множестве $\bar{W}_{U}^{(r)} L_{q}$ формулы (2) достаточно найти моносплайн наименьшего уклонения от нуля на отрезке $[0,1]$ в метрике $L_{p}$ среди всех моносплайнов из $K_{r, \rho}(V)$, а затем воспользоваться $(8)$. 

$\left[{ }^{4}\right]$.

П р и м ч а н и. Доказанная теорема обобщает результат работы

Рассмотрим один частный случай. Пусть $J_{0}, J_{1} \subseteq\{0, \ldots, r-1\}, r-$ четное. На множестве $W^{(r)} L_{q}$ найдем наилучшую формулу вида

$$
\int_{0}^{1} f(x) d x=\sum_{k=1}^{n} \sum_{j=0}^{r-2} B_{k j} f^{(j)}\left(u_{k}\right)+\sum_{j \in J_{0}} b_{j} f^{(j)}(0)+\sum_{j \in J_{1}} c_{j} f^{(j)}(0)+\bar{R}_{n}(f) .
$$

Пусть $R_{r, p}(x)$ - многочлен наименьшего уклонения от нуля на отрезке $[-1,1]$ в метрике $L_{p}$ среди всех многочленов степени $r$ со старшим коэффициентом, равным 1 . При $J \subseteq\{0, \ldots, r-1\}$ через $T_{J, r, p}(x)$ обозначим многочлен наименьшего уклонения от нуля на отрезке [0, 1] в метрике $L_{p}$ среди всех многочленов степени $r$ со старшим коэффициентом, равным 1 , и удовлетворяющих условиям

Обозначим

$$
T^{(j)}(0)=0 \quad(j \in J) .
$$

$$
J_{i}^{c}=\left\{j: j \in\{0, \ldots, r-1\}, r-i-1 \notin J_{i}\right\} \quad(i=0,1) .
$$

Нз теоремы 1 и теоремы 7 из $\left[{ }^{3}\right]$ следует

Т е о ре м а 2. Единственной наилучшей на множестве $W^{(r)} L_{q}$ формулой (10) является формула с узлами

20ิe

$$
u_{k}=\left[\delta_{0}+2(k-1)\right] h \quad(k=1, \ldots, n),
$$

$$
\begin{gathered}
h=1 /\left[2(n-1)+\delta_{0}+\delta_{1}\right], \\
\delta_{0}=\left[R_{r, p}(1) / T_{J_{0}^{c}, r, p}(1)\right]^{\frac{1}{r}}, \quad \delta_{1}=\left[R_{r, p}(1) / T_{J_{1}^{c}, r, p}(1)\right]^{\frac{1}{r}},
\end{gathered}
$$

и весами

$$
\begin{aligned}
B_{1 j} & =\frac{1}{r !}\left[R_{r, p}^{(r-j-1)}(1) h^{j+1}+(-1)^{j} T_{J_{0}^{c}, r, p}(1) u_{1}^{j+1}\right], \\
B_{k j} & =\frac{1}{r !} R_{r, p}^{(r-j-1)}(1) h^{j+1}\left[1+(-1)^{j}\right] \quad(k=2, \ldots, n-1), \\
B_{n j} & =\frac{1}{r !}\left[T_{J_{1}^{c}, r, p}^{(r-j-1)}(1)\left(1-u_{n}\right)^{j+1}+(-1)^{j} R_{r, p}^{(r-j-1)}(1) h^{j+1}\right] \quad(j=0, \ldots, r-2), \\
b_{j} & =\frac{(-1)^{j+1}-u_{1}^{j+1} T_{J_{0}^{c}, r, p}^{(r-j-1)}(0) \quad\left(j \in J_{0}\right),}{r !} \quad\left(j \in J_{1}\right) .
\end{aligned}
$$

Точная оценка ошибки этой формуль есть

$$
\bar{R}_{n}=\frac{M h^{r} R_{r, p}(1)}{r !(r p+1)^{\frac{1}{p}}} .
$$

Эта теорема обобщает некоторые результаты работ $\left[{ }^{3,5-8}\right]$.

П р и еч а н е. Впервые наилучшие на множествах функций квадратурные формулы Маркова были рассмотрены в [ $\left.{ }^{9}\right]$. 


\section{ЛИТЕРА Т У Р А}

1. Никольский С. М., Квадратурные формулы, М., 1974.

2. Н а й м а р к М. А., Линейные дифференциальные операторы, М., 1969.

3. Levin M., Girshovich Y., ENSV TA Toimet., Füüs. Matem., 24, 264 (1975),

4. Л е в н М., Изв. АН ЭССР, Физ. Матем., 21, 449 (1972).

5. Л е в и М., Изв. АН ЭССР, Физ. Матем., 19, 407 (1970).

6. А л хи мов а В. М., Докл. АН СССР, 204, 263 (1972).

7. Й г и А., Изв. АН ЭССР, Физ. Матем., 22, 358 (1973).

8. Лев ин М., Л в в и а М., Изв. АН ЭССР, Физ. Матем., 24, 15 (1975).

9. Л е в и н М., Изв. АН ЭССР, Физ. Матем., 18, 249 (1969).
Таллинский политехнический институт
Поступила в редакцию 10/III 1975

EESTI NSV TEADUSTE AKADEEMIA TOIMETISED. 25. KÖIDE
FOUSIKA * MATEMAATIKA. 1976, NR. 2

ИЗВЕСТИЯ АКАДЕМИИ НАУК ЭСТОНСКОП ССР. ТОМ 25 ФИЗИКА * МАТЕМАТИКА. 1976, № 2

\section{Ю. ГИРШОВИЧ}

\section{ОПТИМАЛЬНЫЕ КВАДРАТУРНЫЕ ФОРМУЛЫ С ФИКСИРОВАННЫМИ УЗЛАМИ}

I. GIRSOVITS OPTIMAALSED FIKSEERITUD SOLMEDEGA KVADRATUURVALEMID

Y. GIRSHOVICH. BEST QUADRATURE FORMULAS WITH FIXED KNOTS

Через $W^{(r)} L_{q}$ (при заданных $M>0$ и $1 \leqslant q \leqslant \infty$ ) обозначим множество всех функций $f(x)$, которые на отрезке $[0,1]$ имеют абсолютно непрерывную производную порядка $r-1$ и удовлетворяют условию

$$
\left\|f^{(r)}\right\|_{L q(0,1)} \leqslant M \text {. }
$$

Пусть задана линейно независимая система функционалов

$$
U_{i}(f)=\sum_{j=0}^{r-1}\left[\alpha_{i j} f^{(j)}(0)+\beta_{i j} f^{(j)}(1)\right] \quad(i=1, \ldots, s),
$$

где $0 \leqslant s \leqslant 2 r$.

Через $W_{U}^{(r)} L_{q}$ обозначим множество всех функций $f(x)$, которые принадлежат множеству $W^{(r)} L_{q}$ и удовлетворяют условиям

$$
U_{i}(f)=0 \quad(i=1, \ldots, s) .
$$

Квадратурную формулу с фиксированными узлами и остатком $R(f)$ будем называть оптимальной $\left[{ }^{1}\right]$ на множестве функций $H$, если ее веса выбраны таким образом, что величина $\left.\sup _{f \in H} \mid R(f)\right\}$ достигает наименьшего значения. Нас будет интересовать задача построения оптимальной на множестве $W_{U}^{(r)} L_{q}$ формулы

$$
\int_{0}^{1} f(t) d t=\sum_{k=1}^{n} \sum_{j \in J_{k}} A_{k j} f^{(j)}\left(x_{k}\right)+R_{n}(f),
$$

где узлы $0 \leqslant x_{1}<\ldots<x_{n} \leqslant 1$ и множества $J_{k} \subset\{0,1, \ldots, r-1\}$ $(k=1, \ldots, n)-$ заданы. 